\title{
Automated Surveillance Track Filter Tuning By \\ Randomized Algorithms
}

\author{
Yvo Boers, Hans Driessen and Nardo Laclé \\ THALES NEDERLAND, Hengelo, The Netherlands \\ yvo.boers@nl.thalesgroup.com
}

\begin{abstract}
Tuning or fine tuning of a tracker system turns out to be a hard job in practice. The main reason for this is that in a practical (surveillance) tracker system there are a lot of design parameters and a lot of competing requirements to be met. This paper provides the user with an algorithm to tune a tracker system automatically and at the same time obtain quantitative results in terms of the optimality of the solution. The theory of randomized algorithms is used to obtain probabilistic statements on the quality of the output of the tuning process. A simplified example illustrates how the developed theory is to be used.
\end{abstract}

Keywords: Target Tracking, Tuning, Randomized Algorithms

\section{Introduction}

This paper deals with automatically tuning a tracker system. Tuning a tracker system can be a hard and tedious job, therefore techniques are being investigated how this process can be automated and how the results of this process can be used and how they should be interpreted. After tuning one also wants to be able to make quantitative statements about the results obtained, also this issue will be treated.

We will develop a method for automated tuning which will result in an almost optimally tuned tracker system w.r.t. performance measures that have been specified by the user. In the proceeding we will explain what we mean by almost optimal.

Recently, probabilistic methods have been employed, predominantly in the field of control system analysis and synthesis. These methods are sample-based methods. The methods have been used to be able to deal with computa- tionally complex problems, sometimes also referred to as NP-hard problems, see e.g. [8], [9], [11], [12], [14], [15] and [16]. These methods are generally referred to as randomized algorithms.

The key idea of randomized algorithms is that the notion of a solution to a problem is slightly changed. Instead of developing an algorithm that solves the problem exactly in all cases, the problem is approximately solved in most of the cases. The notions, "approximately" and "most of the cases" are being quantified and a probability that the algorithm fails is specified. Also explicit bounds for the number of required samples are given. By settling for solutions in terms of probabilistic statements, one is able to solve a much wider variety of problems.

\section{Optimization by randomized algo- rithms}

In this section we will give some background on the Randomized Algorithms (RA's). In order to relate the general theory to the specific problem of tuning a tracker system in the proceeding the space $\Phi$ can be interpreted as the space of 'tuning parameters'. The space $\Theta$ is to be interpreted as the space of (measurement) noise realizations. A criterion function depending on a tuning parameter vector $\phi \in \Phi$ is minimized in a probabilistic sense, this criterion function is obtained by taking the (empirical) average over $\Theta$, i.e. over (a lot of) noise realizations for the performance function that has been specified by the user. 


\subsection{A special type of minimum}

In a RA approach, the notion of a minimum of a function is given a probabilistic meaning. Instead of exactly minimizing a function, in a probabilistic setting one has to be satisfied with nearly minimizing a function.

We consider a type of minimum that has been introduced in [11].

Let $\Phi$ be a set and suppose $f: \Phi \rightarrow \mathbb{R}$ is a function. Let

$$
f^{*}=\inf _{\phi \in \Phi} f(\phi)
$$

We will now give the definition of a probably approximate near minimum.

\section{Definition 2.1}

Suppose $Q$ is a probability measure on $\Phi$ and $\epsilon>0,0<$ $\alpha<1$ are given numbers. A number $f^{0} \in \mathbb{R}$ is called a probably approximate near minimum of $f$ to accuracy $\epsilon$ and level $\alpha$, if

$$
f^{0} \geq f^{*}-\epsilon
$$

and in addition

$$
Q\left\{\phi \in \Phi \mid f(\phi)<f^{0}-\epsilon\right\} \leq \alpha
$$

Definition 2.1 states that there exists a set $S=\left\{\phi \in \Phi \mid f(\phi)<f^{0}-\epsilon\right\}$, with $Q(S) \leq \alpha$ such that

$$
f^{*}-\epsilon \leq f^{0} \leq \inf _{\phi \in \Phi \backslash S} f(\phi)+\epsilon .
$$

In words, if we exclude the small (with respect to the measure Q) set $S, f^{0}$ is not more than $\epsilon$ away from the actual infimum of $f$.

\subsection{Determining the empirical mean}

Let $s: \Theta \rightarrow[0,1]$ be a measurable function with respect to the measure $P$. The expectation of $s$ over $\Theta$ is

$$
E_{\Theta}(s)=\int_{\Theta} s(\theta) d P
$$

To approximate this quantity, $M$ samples $\theta_{1}, . ., \theta_{M}$ are drawn according to $P$ and the empirical mean is determined as:

$$
\hat{E}(s, \boldsymbol{\theta}):=\frac{1}{M} \sum_{j=1}^{M} s\left(\theta_{j}\right)
$$

where $\boldsymbol{\theta}:=\left[\theta_{1}, \ldots, \theta_{M}\right]^{T} \in \Theta^{M}$.

Denote by $P^{M}$ the probability measure on the set $\Theta^{M}$ that is induced by the probability measure $P$ on the set $\Theta$.

Now Hoeffdings inequality (for more details see [10] and [11]) states that $\forall \epsilon>0$ we have:

$$
P^{M}\left(\boldsymbol{\theta} \in \Theta^{M}:\left|\hat{E}(s, \boldsymbol{\theta})-E_{\Theta}(s)\right|>\epsilon\right) \leq 2 e^{-2 M \epsilon^{2}}
$$

In words, inequality (7) states that once $M$ samples $\theta_{1}, . ., \theta_{M}$ have been drawn from $\Theta$, according to $P$, the probability that the empirical mean differs more then $\epsilon$ from the exact value of the mean is smaller then $2 e^{-2 M \epsilon^{2}}$.

So in order to estimate the mean $E_{\Theta}(s)$ to an accuracy $\epsilon$ and with a confidence of at least $1-\delta$, one should have $\delta \leq 2 e^{-2 M \epsilon^{2}}$ or, equivalently

$$
M \geq \frac{1}{2 \epsilon^{2}} \ln \frac{2}{\delta} .
$$

A nice feature of this bound is the fact that it is independent of the dimension of the underlying set $\Theta$. This fact is also exploited in Monte Carlo simulation.

\subsection{An algorithm to produce a probably ap- proximate near minimum}

In this section we will provide an algorithm that produces a probably approximate near minimum, see definition 2.1 .

Define

- Sets $\Theta \subset \mathbb{R}^{n}$ and $\Phi \subset \mathbb{R}^{k}$.

- Probability measures $P$ and $Q$ on $\Theta$ and $\Phi$, respectively.

- A measurable function $h: \Theta \times \Phi \rightarrow[0,1]$.

- $f(\phi):=E_{\Theta}[h(\theta, \phi)]$

- A level parameter $0<\alpha<1$. 
- An accuracy parameter $0<\epsilon<1$.

- A confidence parameter $0<\delta<1$.

\section{Algorithm 2.2}

\section{STEP 1}

set

$$
\begin{gathered}
N \geq \frac{\ln \frac{2}{\delta}}{\ln \frac{1}{1-\alpha}} \\
M \geq \frac{1}{2 \epsilon^{2}} \ln \frac{4 N}{\delta}
\end{gathered}
$$

\section{STEP 2}

Generate samples $\phi_{1}, . ., \phi_{N} \in \Phi$ according to $Q$ and $\theta_{1}, . ., \theta_{M} \in \Theta$ according to $P$.

\section{STEP 3}

Define the empirical means

$$
\hat{f}_{i}:=\frac{1}{M} \sum_{j=1}^{M} h\left(\theta_{j}, \phi_{i}\right), i=1, . ., N
$$

and set

$$
\hat{f}_{0}:=\min _{i \in\{1, . ., N\}} \hat{f}_{i}
$$

Now the following theorem can be formulated.

\section{Theorem 2.3}

With confidence $1-\delta, \hat{f}_{0}$ is a probably approximate near minimum of $f$ to accuracy $\epsilon$ and level $\alpha$.

For a proof of this theorem we refer to [11] or [16].

By applying algorithm 2.2 one also obtains the values $\phi^{0} \in \Phi$ for which the probably approximate near minimum $\hat{f}_{0}$ is achieved.

$\phi^{0}$ is obtained as

$$
\phi^{0}:=\arg \min _{i \in 1, \ldots, N} f\left(\phi_{i}\right)
$$

\section{Tuning a track filter: example}

In this example we will tune a type of track filter that has been proposed in [17]. The system setup will be the exact same as the one used in [17]. For the sake of completeness we will list some relevant characteristics of this track filter, i.e. the Multiple Model Multiple Hypothesis Filter (MMMHF).

The system under consideration is that of a long range surveillance radar.

The relevant characteristics of the radar system are listed in table 1.

\begin{tabular}{|l|l|}
\hline \multicolumn{2}{|c|}{ characteristics } \\
\hline \hline description & value \\
\hline rotation speed & $12 \mathrm{rpm}$ \\
\hline range accuracy: $\sigma_{\text {range }}$ & $20 \mathrm{~m}$ \\
\hline bearing accuracy: $\sigma_{\text {bearing }}$ & $8 \mathrm{mrad}$ \\
\hline elevation accuracy: $\sigma_{\text {elevation }}$ & $8 \mathrm{mrad}$ \\
\hline doppler accuracy: $\sigma_{\text {doppler }}$ & $10 \mathrm{~m} / \mathrm{s}$ \\
\hline
\end{tabular}

Table 1: Relevant radar characteristics

The target behavior can be modeled in a generic way by:

$$
s(k+1)=F(s(k), t(k), m(k))+
$$

$$
+G(s(k), t(k), m(k)) w(k, m(k)), \quad k \in \mathbb{N}
$$

$z(k)=H(s(k), t(k), m(k))+v(k, m(k)), \quad k \in \mathbb{N}$

where $F, G$ and $H$ are smooth functions and

- $s(k) \in \mathbb{R}^{n(m(k))}$ is the kinematic state of the system.

- $m(k) \in \mathbf{M} \subset \mathbb{N}$ is the modal state of the system.

- $z(k) \in \mathbb{R}^{p(m(k))}$ is the measurement.

- $t(k) \in \mathbb{R}$ is time.

- $w(k)$ is the process noise and $d_{w(k, m(k))}(w)$ is the probability distribution of the process noise.

- $v(k)$ is the measurement noise and $d_{v(k, m(k))}(v)$ is the probability distribution of the measurement noise. 
The MMMHF is a three model filter

The first mode has the following state:

$$
s(k)=\left[x(k), y(k), z(k), v_{x}(k), v_{y}(k)\right]^{T}
$$

, i.e. the cartesian position and the horizontal speeds. This mode corresponds to a constant velocity model, see [4].

The second mode has the following state:

$$
s(k)=\left[x(k), y(k), z(k), v_{x}(k), v_{y}(k), v_{z}(k), \omega(k)\right]^{T}
$$

, i.e. the cartesian position, the cartesian velocity and an angular velocity. This mode corresponds to a coordinated turn model, see [4]. Note that for this model the dynamics are nonlinear.

And the third mode has the state:

$s(k)=\left[x(k), y(k), z(k), v_{x}(k), v_{y}(k), v_{z}, a_{x}(k), a_{y}(k), a_{z}(k)\right.$

, i.e. the cartesian position, the cartesian velocity and the cartesian acceleration. This mode corresponds to a constant acceleration model, see [4].

The Markov transition matrix is given by:

$$
\left(\begin{array}{ccc}
0.96 & 0.02 & 0.02 \\
0.02 & 0.96 & 0.02 \\
0.02 & 0.02 & 0.96
\end{array}\right)
$$

and the measurements are given by:

$$
\begin{aligned}
& H_{1}(s)=\sqrt{x^{2}+y^{2}+z^{2}} \\
& H_{2}(s)=\operatorname{atan}(y / x) \\
& H_{3}(s)=\operatorname{atan}\left(z / \sqrt{x^{2}+y^{2}}\right) \\
& H_{4}(s)=\frac{x \cdot v_{x}+y \cdot v_{y}+z \cdot v_{z}}{\sqrt{x^{2}+y^{2}+z^{2}}}
\end{aligned}
$$

As reported in [17], there are still 'free' tuning parameters in the MMMHF. These parameters are:

- The maximum hypothesis depth or hypothesis history, $\in\{1,2, \ldots, 10\}$.

- The Maximum number of hypotheses that will be maintained, $\in\{1,2, \ldots, 8\}$.
- A threshold, below which hypotheses are pruned $\epsilon$ $[0.001,0.1]$.

The goal now is to find parameter settings that are optimal for a certain set of scenarios. By optimal we mean optimal in the sense of definition 2.1.

We have scenarios including a straight flying target, a sharply (4g) maneuvering target and a target in a soft maneuver $(1 \mathrm{~g})$, see figures 1 and 2.

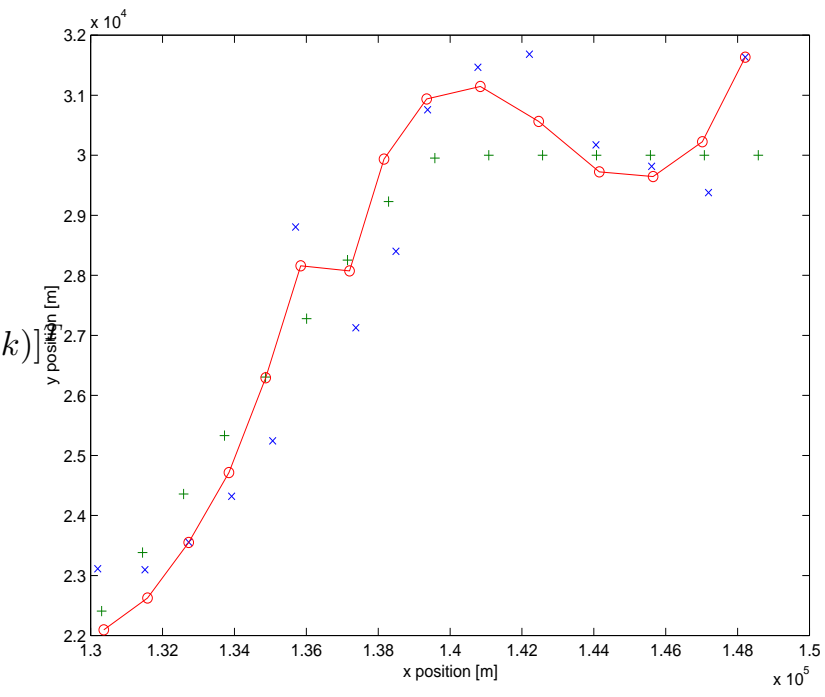

Figure 1: Straight and sharp turn scenario. +=true, $\mathrm{x}=$ measurement and $\mathrm{o}=$ filtered

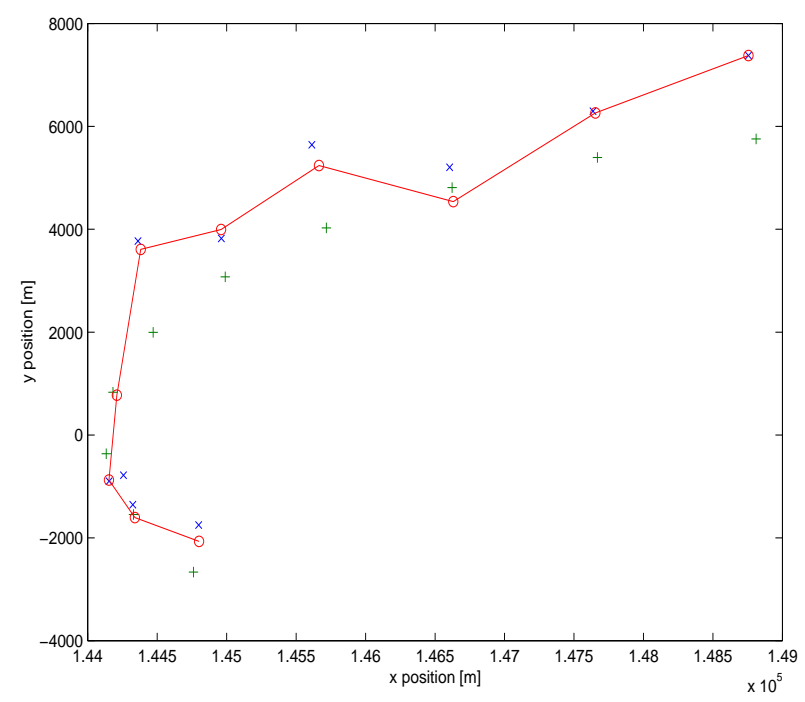

Figure 2: Soft turn scenario. $+=$ true, $x=$ measurement and $\mathrm{o}=$ filtered

The performance criterion we will define is a tradeoff between track accuracy and computational load. 
For one realization of a scenario with a certain choice of parameter setting the criterion function $h$, see algorithm 2.2 , is given by:

$$
h(., .):=0.9 \frac{\gamma_{1} M S E}{\gamma_{1} M S E+1}+0.1 \frac{\gamma_{2} M N O F H}{\gamma_{2} M N O F H+1}
$$

In equation (16) MSE is the mean squared tracking error, MNOFH is the mean number of hypotheses processed for a certain realization, $\gamma_{1}=0.001$ and $\gamma_{2}=0.01$ are scaling parameters.

Clearly the function $h$ ranges in the interval $[0,1]$.

Taking the (empirical) mean of $h$ over realizations of measurement noise will produce the function $f$, see algorithm 2.2.

The goal is now to minimize $f$, in the sense of definition 2.1 , w.r.t. the tuning parameter.

We want to have a confidence $1-\delta$ of 0.99 , a level of probability $\alpha=0.01$ and an accuracy of $\epsilon=0.05$, this implies that we have to test $N=500$ parameter values over $M=2500$ realizations of the scenarios. Furthermore the probability measures $P$ and $Q$, see algorithm 2.2, have been chosen uniform.

Applying algorithm 2.2 results in an optimal hypothesis depth of 4 and an optimal maximum number of hypotheses to be maintained of also 4 , the optimal threshold turns out to be 0.0043 .

Note that we have found from different experiments that the criterion is quite insensitive to the threshold, but every different run of the algorithm resulted in the same values for both the hypothesis depth and the maximum number of hypothesis to be maintained.

We also note that the 'optimal' number of 4 , for the hypothesis depth, is in accordance with the results/conjectures, that were reported in [6] and [7], where the hypothesis depth is also referred to as internal degrees of freedom.

\section{Discussion and implementational issues}

We have chosen the criterion function in the example of the form

$$
\frac{x}{1+x}
$$

where $x$ is a scaled version, scaled by $\gamma_{1,2}$ in the example, of the actual performance measures MSE and MNOFH. This form of the function has been chosen to make sure that the performance function to be minimized is in the interval $[0,1]$.

\section{Remark 4.1}

Note that the above choice of the form of the criterion function is just a choice and that other functions ranging in the interval $[0,1]$ may also be used. Another example of such a function could be to set it to zero if a requirement is met and set it to one if a requirement is not met.

The scaling parameters $\gamma_{1,2}$ are chosen such that the criterion function is 'sensitive' to a change in design/tuning parameters. Looking at the function

$$
\frac{x}{1+x}
$$

in figure 3 we see that at 'small' values of $x(x \in[0,4])$ the sensitivity is quite high, so we scale $M S E$ and $M N O F H$ by the scaling parameters $\gamma_{1,2}$ to make sure that both $\gamma_{1} M S E$ and $\gamma_{2} M N O F H$ are small.

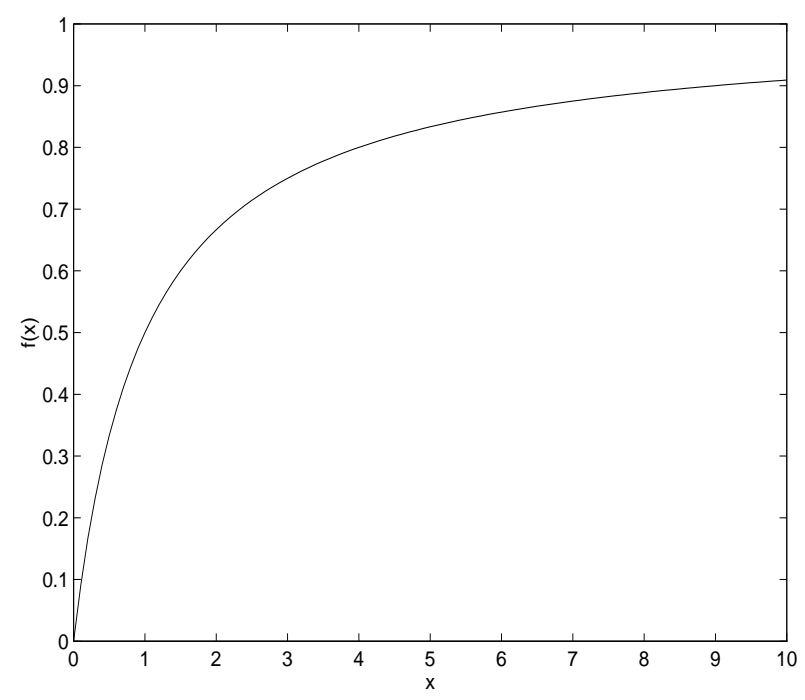

Figure 3: Function $\frac{x}{1+x}$

Furthermore the weights that determine the tradeoff between accuracy and computational load (0.9 and 0.1) have 
also been determined on an experimental basis, i.e. running the algorithm for smaller numbers $N$ and $M$.

We also like to stress the fact that although the theory and the algorithm, presented in this paper, provide a way of automated tuning that can be applied in the design of a tracking system and provides the user with quantitative statements on the optimality of the design, it is still required that the user applies common sense and a priori knowledge in the process of designing a suitable/sensible criterion function.

\section{Conclusions}

In this paper we have presented a new algorithm that can be used for the tuning process of a tracker system. The algorithm provides the user with a probabilistic statement on the quality of the solution. The quality that can be guaranteed is directly related to computational load that is spent on the tuning process. In an example the theory and design of a sensible criterion function has been illustrated.

\section{References}

[1] N.J. Gordon, D.J. Salmond and A.F.M. Smith. Novel approach to nonlinear/non-Gaussian Bayesian state estimation. IEEE Proceedings- $F$, vol. 140, no.2, 1993.

[2] E. Mazor, A. Averbuch, Y. Bar-Shalom and J. Dayan. Interacting multiple model methods in target tracking: a survey. IEEE Transactions on Aerospace and Electronic Systems, vol. 34, pp. 103-123, 1998.

[3] H.A.P. Blom Bayesian Estimation for Decision - Directed Stochastic Control, PhD Thesis, Delft University of Technology, 1990.

[4] S. Blackman and R. Popoli, Design and Analysis of Modern Tracking Systems, Artech House, Norwood, MA, 1999.

[5] Y. Bar-Shalom and X.R. Li. Estimation and Tracking Principles, Techniques and Software, Artech House, Norwood, MA, 1993.

[6] W. Koch. Adaptive parameter control for phase-array tracking. In Proceedings of the conference on Signal and Data Processing of Small Targets. Denver, CO, 1999.
[7] W. Koch. Generalized smoothing for multiple model/multiple hypothesis filtering: Experimental results. In Proceedings of the European Control Conference. Karlsruhe, Germany, 1999.

[8] P. Khargonekar and A. Tikku. Randomized algorithms for robust control analysis and synthesis have polynomial complexity. In Proceedings of the 35th Conference on Decision and Control. Kobe Japan, 1996

[9] R. Tempo and E.W. Bai and F. Dabbene. Probabilistic robustness analysis: Explicit bounds for the number of samples. In Proceedings of the 35th Conference on Decision and Control. Kobe Japan, 1996

[10] W. Hoeffding. Probability inequalities for sums of bounded random variables. J. Emer. Statist. Assoc., vol. 58, pp. 13-30, 1963.

[11] M. Vidyasagar. Statistical learning theory and its applications to randomized algorithms for robust controller synthesis Plenary Lectures and Mini-Courses at the European Control Conference Brussels, Belgium, 1997.

[12] M. Vidyasagar. A theory of learning and generalization. Springer, London, 1997.

[13] Y. Boers. On the number of samples to be drawn in particle filtering.In proceedings of the IEE colloquium on Target Tracking, London, UK, 1999

[14] Y. Boers, S. Weiland and A.A.H. Damen. Average $\mathcal{H}_{2}$ control by randomized algorithms. To appear in the International Journal of Control., 2002.

[15] Y. Boers and S. Weiland and A.A.H. Damen. Average $\mathcal{H}_{2}$ performance design by randomized algorithms In Proceedings of the 37st IEEE Conference on Decision and Control Tampa, FL, 1998.

[16] Y. Boers. Average Performance Control for Systems With Parametric Uncertainty. Technical University of Eindhoven Library Press, ISBN-90-386-1549-X, 1999.

[17] J.N. Driessen and Y. Boers. A multiple model multiple hypothesis filter for tracking maneuvering targets. In proceedings of the Conference on signal and data processing of small targets, San Diego, CA, 2001. 